\title{
Grundlagen zu Tinnitus: Diagnostik und Therapie
}

\author{
Gerhard Goebel ${ }^{1}$ und Ulrich Büttner ${ }^{2}$ \\ ${ }^{1}$ Medizinisch-Psychosomatische Klinik Roseneck, Prien am Chiemsee \\ 2Neurologische Klinik der Universität München, Klinikum Großhadern
}

psychoneuro 2004; 30 (6): 322-329

Tinnitus beschreibt die falsche Wahrnehmung von Tönen und Geräuschen ohne einen entsprechenden äußeren Reiz. Es ist ein häufiges Phänomen, was praktisch jeder erfährt. Ca. 4\% der deutschen Bevölkerung geben ein chronisches Ohr-Kopfgeräusch an (Tinnitus für mehr als $1 \mathrm{Jahr}$ ), wobei ca. 15\% davon sehr beeinträchtigt ist (dekompensierter Tinnitus). Klinisch unterscheidet man subjektiven und objektiven Tinnitus. Letzteres kommt sehr selten vor: Es handelt sich dabei um reale Geräusche (vaskulären Störungen, Gaumensegel-Myoklonus, offeneTuba Eustachii u.a.), die u.U. mit einem Stethoskop über dem Kopf oder Gehörgang gehört werden. Es ist daher kein Tinnitus im engeren Sinne und besser mit „Körpergeräusch“ zu umschreiben. Der subjektive Tinnitus ist in den meisten Fällen mit einer Hörstörung verbunden, tritt aber auch mit Schwindel in Form des Morbus Menière auf. Nur bei 10-20\% besteht keine begleitende Hörminderung. Es gibt nur wenige effektive Akutbehandlungsmöglichkeiten. Im chronischen Stadium sind Behandlungsverfahren zu favorisieren, die eine Tinnitusbewältigung anstreben (z.B. Tinnitus-Retraining-Therapie TRT), Nutzung von Geräuschen incl. großzügigem Einsatz von Hörgeräten zu Teilmaskierung des Tinnitus, bei ausgeprägtem Leiden stationäre Psychotherapie in spezialisierten Kliniken.

$\mathrm{T}$ innitus ist der medizinische Fachausdruck für alle Arten von Ohr- und Kopfgeräuschen unabhängig von deren Ursachen. Vor allem mit einem akuten Beschwerdebild in Verbindung mit Hörverlust oder Schwindel kommen die Patienten in die ärztliche Behandlung.

\section{- Epidemiologie}

Es ist schwierig, die Häufigkeit des Vorkommens zu bestimmen, da wohl jeder Mensch das Phänomen Tinnitus kennt. Nach Ergebnissen einer 1998 durchgeführten repräsentativen Erhebung der Deutschen Tinnitus-Liga (2) kommt es jährlich bei ca. 10 Millionen Deutschen zu Tinnitus (Neuerkrankungsrate), der bei ca. 250000 in eine chronische Form übergeht. Ca. drei Millionen Erwachsene in Deutschland (4\% der Bevölkerung) sind somit von chronischem Tinnitus betroffen (Punktprävalenz). Die Ohrgeräusche werden von $37 \%$ nur bei Stille wahrgenommen, bei $44 \%$ lässt sich der Tinnitus durch Umgebungsgeräusche überdecken, wohingegen er sich in
17\% der Fälle selbst durch starken Lärm nicht übertönen lässt.

\section{- Prognose}

Bei akutem Tinnitus beträgt die Spontanheilungsrate $60-80 \%$. Bei subakutem (länger als 3 Monate) bzw. chronischen Tinnitus (länger als ein Jahr) sind die Chancen einer Spontanheilung gering. Verläufe mit quälendem Tinnitus über viele Jahre sind nicht selten. Bei Patienten mit chronischem Tinnitus nimmt dieser nur bei $8-13 \%$ der Patienten im Laufe der Zeit zu. In der Regel wird er nach ca. 18 Monaten leiser bzw. verliert seinen zentralen Stellenwert. Innerhalb von zehn Jahren wird er bei mehr als der Hälfte der Betroffenen nur noch intermittierend oder gar nicht mehr wahrgenommen (1).

\section{Pathophysiologie \\ des Tinnitus}

Ohrgeräusche sind ein Symptom des Hörsystems. Ähnlich dem optischen System, das bei Störungen nur mit Sehminderung, subjektiven

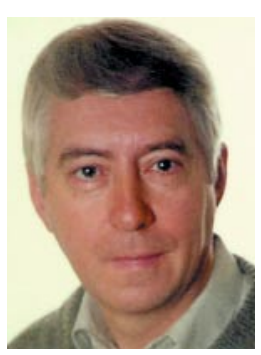

Ulrich Büttner
Lichtblitzen oder Lichtempfindlichkeit reagieren kann, führen Störungen des eigentlichen Hörsystems ausschließlich zu Hörminderung, Tinnitus oder Geräuschüberempfindlichkeit. In einer Vielzahl von Modellen wurde versucht, die Entstehung von Tinnitus auf den verschiedenen Ebenen des auditiven Systems darzustellen, um mögliche therapeutische Vorgehensweisen abzuleiten. Ursachen, die in der Cochlea (Innenohr), dem Hörnerv und der zentralen Hörbahn liegen (Abb. 1), können nur nach Anamnese- und Befunderhebung hypothetisch zugeordnet werden. Festlegungen auf Pauschalhypothesen wie Durchblutungsstörung oder Stress werden der Differenziertheit des Tinnitus nicht gerecht (5).

Für die primäre Tinnitusentstehung sind Schäden der inneren Haarzellen (IHZ) und äußeren Haarzellen (ÄHZ) in der Cochlea sowie Störungen des Signaltransfers im Synapsenbereich von wesentlicher Bedeutung (Abb. 2): Durch Schädigung oder komplette Zerstörung der ÄHZ (z.B. Lärmschädigung, ototoxische Einflüsse etc.) wird der dämpfende und modulierende Effekt auf die IHZ gestört und kann somit neben einer Einschränkung differenzierter Hörvorgänge zu einer Geräuschempfindlichkeit und Tinnitus führen (Rekruitment). Bei zusätzlicher Schädigung der IHZ kann die Umwandlung von Schallwellen in elektrische Nervenimpulse nur noch teilweise realisiert werden einhergehend mit Hörminderung und fehlerhaften Entladungen der Haarzellen (Transformationstinnitus; 15). 
Es kann sich aber auch um pathologische Prozesse im Bereich der Synapsen (exzitotoxischer Tinnitus) sowie um Spontanentladungen von Fasern des 8. Hirnnervs selbst (z.B. Akustikusneurinom) und schließlich um Spontanentladungen oder Versagen von Hemmfunktionen zentraler Hörbahnbereiche handeln (primär-zentraler Tinnitus). Hiermit gut vereinbar sind neuere Untersuchungen, nach denen transkranielle Kortexstimulation über dem temporo-parietalen Kortex zu einer vorübergehenden Tinnitusreduktion führt (14).

Analog zur Trigeminusneuralgie wird auch die Möglichkeit von ephaptischen Erregungen benachbarter, teilweise demyelinisierter Axone durch Gefäßschlingenkompression des Nervenaustrittes am Hirnstamm diskutiert (4).

Weitere Prozesse der Tinnitusauslösung sind Störungen des Ionengleichgewichts in den verschiedenen Medien der Cochlea, Resorptionsstörungen, Osmolaritätsänderungen, Endolymphhydrops (Abb. 3), Durchblutungsstörungen der Stria vascularis sowie eine unausgewogene Aktivität der Nervenfasern $(4,7,15)$.

Die bisherigen Modellvorstellungen der peripheren und primärzentralen Tinnitusgenerierung gingen davon aus, dass über die pathologische Hörnervenaktivität bzw. eine veränderte Spontanaktivität das auditorische System irritiert wird und der Tinnitus auf der Hörrinde als abnormes Muster abgebildet wird. Zwischenzeitlich werden auch Plastzitätsvorgänge im auditiven Kortex vermutet, die unabhängig von den ursprünglichen peripheren Ursachen zu einer Verselbstständigung im Sinne einer „Zentralisierung“ führen und damit zu den eigentlichen Tinnitusgeneratoren werden. Der Tinnitus wäre damit das Pendant zum Phantomschmerz (Phantom-Tinnitus sekundär-zentraler Tinnitus) $(9,15)$.

Die Kombination mehrerer Tinnitustypen ist grundsätzlich möglich und gilt insbesondere für den sekundär-zentralen Tinnitus. Vergegenwärtigt man sich die hier skizzierte neurophysiologische Dif- ferenziertheit des Hörsystems, erscheint besonders beim chronischen matogenen und psychogenen oder peripheren und zentralen Tinnitus problematisch (5).

\section{Tinnitusursachen und deren Therapie}

Mehr als zwei Drittel der Tinnitusfälle lassen sich auf Innenohrschwerhörigkeit als chronisch-progrediente Form oder als Lärmschwerhörigkeit, gefolgt von Morbus Menière und Hörsturz zurückführen (Abb. 3; 4, 7). Bei 25\% bis $40 \%$ der Fälle bleibt die Ursache unklar („idiopathischer Tinnitus“). Zur umfassenden Analyse der verschiedenen Tinnitusursachen kann das evaluierte „Strukturierte Tinnitus-Interview (STI)“ herangezogen werden (10). Zur Graduierung des Tinnitusschweregrades dient der in Deutschland etablierte „Tinnitus-Fragebogen (TF)“” von Goebel \& Hiller (6).

\section{Tinnitus mit Hörstörungen}

- Bei Innenohrschwerhörigkeit: Generell ist davon auszugehen, dass ein Hörschaden gleich welcher Art eine Prädisposition für eine veränderte Aktivität zentraler Hörbahnabschnitte darstellt ren kann. Etwa zwei Drittel von Personen mit Schwerhörigkeit geben Tinnitus an, von denen wiederum etwa $20 \%$ das Ausmaß Tinnitus die Dichotomisierung in sound sich als Tinnitus manifestie-

eines dekompensierten Tinnitus aufweisen. Bei einem Drittel der Schwerhörigen lassen sich familiäre Dispositionen eruieren (hereditäre Schwerhörigkeit; 7). Therapie: Eine frühzeitige Versorgung mit Hörgeräten ist im Hinblick auf eine langfristig erfolgreiche Kompensierung des Tinnitus indiziert $(4,9)$

- Bei Otosklerose: Otosklerose ist gekennzeichnet durch eine entzündlich bedingte fortschreitende Immobilität des Steigbügels (Schallleitungsschwerhörigkeit) und ist in 70\% mit Tinnitus vergesellschaftet. Therapie: Der Tinnitus sistiert nach mikrochirurgischer Revision (Stapesplastik) in ca. 30-50\% der Fälle, er kann sich aber auch operativ bedingt verschlechtern

- Bei Hörsturz: Der idiopathische Hörsturz ist charakterisiert als ein akuter, aus scheinbarem Wohlbefinden heraus auftretender einseitiger Hörverlust im Sinne einer Schallempfindungsschwerhörigkeit. Tinnitus kommt dabei in 70\% der Fälle vor (4). Therapie: Spontanremissionen sind besonders bei Tieftonhörstürzen häufig. Zur Behandlung wird 6\%-iges HAES mit Prednisolon verwandt (13)

- Bei Schädel-Hirn-Trauma: Der meist hochfrequente Tinnitus dauert meist nur einige Stunden. Bei Innenohrläsion kann er jahre-

\section{Abb. 1 Das Ohr}

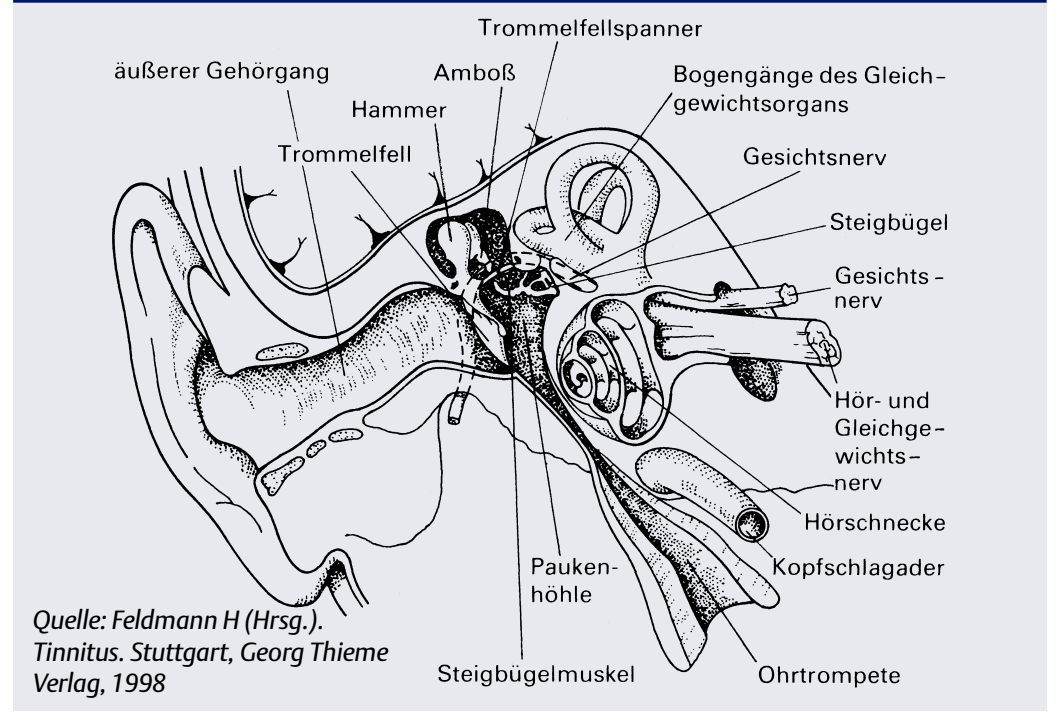


lang anhalten. Therapie: Unter der Annahme eines gleichzeitigen Knalltraumas kann eine Therapie mit Prednisolon erwogen werden

- Bei Lärm-/Knalltrauma infolge akuter oder chronischer Lärmexposition: Therapie: Beim akuten Lärmtrauma wird über therapeutische Effekte von Prednisolon in Kombination mit hyperbarer Sauerstofftherapie (HBO) berichtet (13)

- Bei Akustikusneurinom: Langsam fortschreitende oder fluktuierend auftretende einseitige Hörminderung mit intermittierendem hochfrequenten Tinnitus ist oft der erste Hinweis auf ein Akustikusneurinom. Viele Neurinome manifestieren sich initial nur mit einem Tinnitus. Therapie: Operation oder radiochirurgische Therapie. Ziel der Therapie ist primär die Funkti- onserhaltung des 7. und 8. Hirnnerven. Nicht immer sistiert als „Nebeneffekt“ der Tinnitus (s. unten)

- Nach ototoxischen Medikamenten oder Giften: Aminoglykoside (bes. Kanamycin, Streptomycin, Neomycin, Bykomycin), Cisplatin, Schwermetalle. Therapie: Absetzen der Medikamente, Eliminierung der Giftstoffe. Bleibende Schäden sind möglich.

\section{Tinnitus ohne Hörstörung}

Bei vielen Patienten, die über Tinnitus klagen, lassen sich keine relevanten Funktionsstörungen des Gehörs nachweisen. Bei bis zu 50\% der Betroffenen geht eine Geräuschempfindlichkeit (Hyperakusis) voraus (8).

Therapie: Im akuten Stadium wird der HNO-Arzt je nach Belästigungsgrad eine Infusionsbehandlung

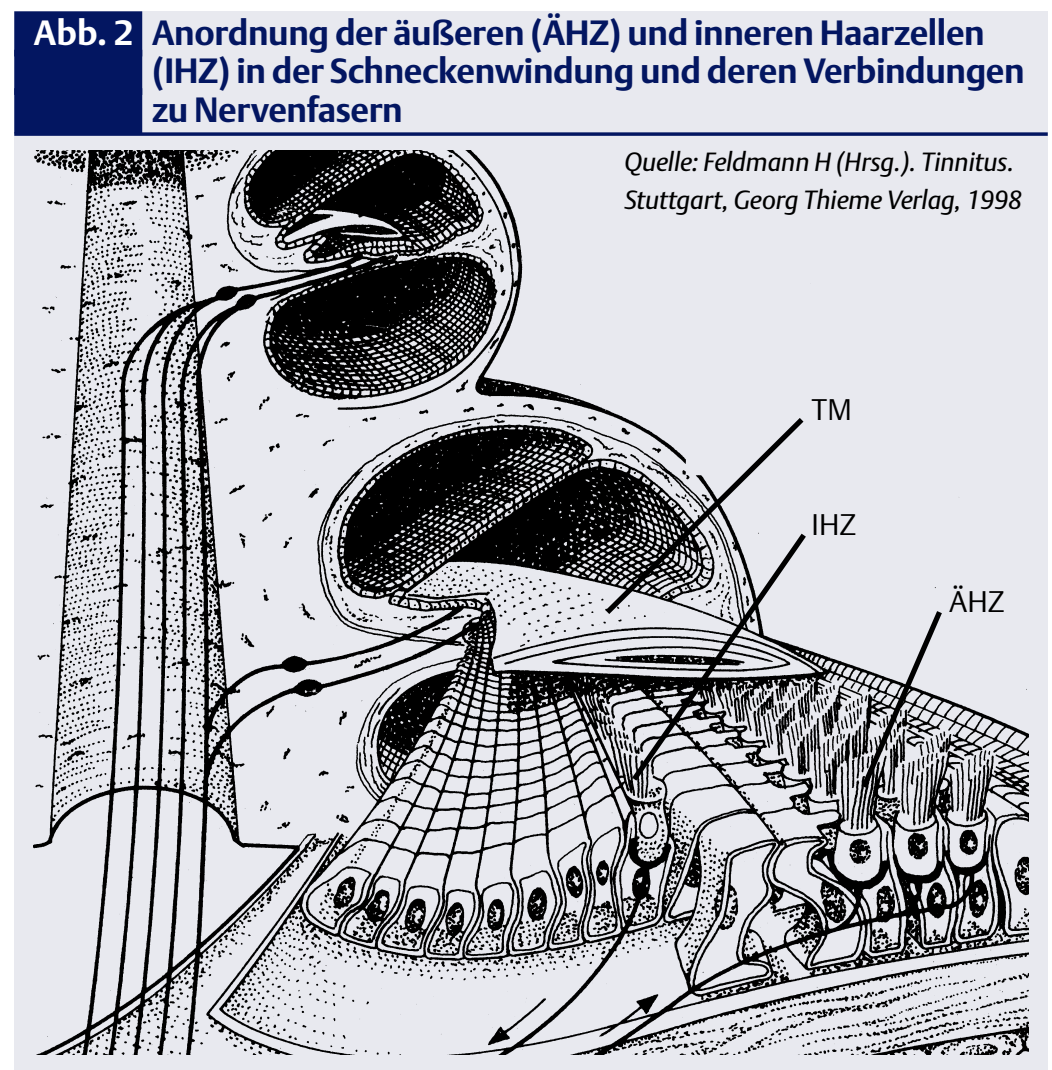

Die inneren Haarzellreihen (IHZ) stehen über Afferenzen mit dem Gehirn in Verbindung. Alles, was „hörbar“ aus dem Ohr kommt, wird also über diese Sinneszellen vermittelt. Die drei äußeren Haarzellreihen (ÄHZ) unterliegen überwiegend efferenten Verschaltungen. Durch ihre Kontraktilität reagieren sie auf eine Vielfalt von Einflüssen des Hörsystems: Sie modulieren „vor Ort“ im Zusammenspiel mit der ihr aufliegenden Tektorialmembran (TM) die Empfindlichkeit der Cochlea.

wie bei Hörsturz anbieten, die in Absprache mit dem Patienten festgelegt werden sollte. Dabei ist in Anbetracht fehlender Erklärungen des Tinnitus bei einem entsprechend informierten Patienten auch das Zuwarten ohne Behandlung kein Fehler.

Funktionsstörungen der Halswirbelsäule oder der Kiefer-Kaumuskulatur

Häufig finden sich durch traumatische oder chronische Muskelverspannungen bedingte degenerative Halswirbelsäulenveränderungen oder Muskelverspannungen, die über efferente Verschaltungen mit der Hörbahn unterschiedliche Arten von einseitigem Tinnitus bedingen oder einen bestehenden Tinnitus sekundär verstärken (zervikogener Tinnitus; Biesinger 2001). Dies gilt auch für Störungen im Kiefergelenkbereich und das häufig verbreitete Zähneknirschen (stomatognathogener Tinnitus; 7).

Therapie: fachärztliche Behandlung, Krankengymnastik, Relaxationsübungen.

\section{Als Nebenwirkung von Pharmaka}

Tinnitus ist eines der Symptome, die häufig als Nebenwirkung vieler Medikamente auf den Beipackzetteln der Medikamente aufgeführt werden. In der Regel reversibel und dosisabhängig kann Tinnitus auftreten bei Chinidin, Salizylaten, Indometacin, Carbamazepin, Propranolol, Salbutamol, Levodopa, Aminophyllin, Koffein, Tetrazyklin, Doxyzyklin, Antidepressiva, etc. (ausführliche Übersicht 4). Dies bedeutet nicht, dass diese Medikamente Tinnitusbetroffenen vorenthalten werden sollten. Die Patienten sind darüber aufzuklären. Bei Lauterwerden des Tinnitus ist unter Abwägen der medizinischen Umstände ein Absetzversuch zu diskutieren.

\section{Tinnitus mit Drehschwindel}

Die Trias rezidivierender Tinnitus mit Hörsturz und Drehschwindelanfällen entspricht dem klassischen Bild eines M. Menièreanfalles. Fast immer besteht auch ein Druckgefühl auf dem befallenen Ohr. Oft kündigt sich der Anfall auf der betroffenen 
Seite mit dem Druckgefühl an und bei chronischem Tinnitus - mit einem Lauterwerden des Ohrgeräusches sowie einer Dysakusis (verzerrtes Hören). Selten beginnt die Menière'sche-Krankheit mit dem Vollbild der typischen Symptome, vielmehr findet sich in der Frühphase nur eines der Symptome, was die Diagnosestellung zum Krankheitsbeginn erschwert. Ebenso gelingt es in den beschwerdefreien und symptomlosen Intervallen anfänglich nicht, typische Krankheitszeichen nachzuweisen. Im späteren Stadium sind dann bleibende Funktionseinschränkungen im vestibulären und cochleären System nachweisbar.

Ätiologisch handelt es sich um einen endolymphatischen Hydrops des Innenohrs (Scala tympani; siehe Abb. 3), der - bei Einreißen der Reisnerschen Membran - durch Vermischung der lymphatischen Flüssigkeitskomponenten zur Reizung des betroffenen Vestibularapparates und damit zum Drehschwindelanfall führt. Entsprechend sieht man im Anfall in der Regel horizontal rotierenden Nystagmus. Begleiterscheinungen des Anfalles sind gerichtete Fallneigung, Blässe, Schweißausbruch bis hin zu Diarrhö, Nausea und Erbrechen. Der Schwindel sistiert üblicherweise innerhalb 24 Stunden (11).

Therapie: Im akuten Stadium: Antiverginosa Dimehydronat (Vomex ${ }^{\circledR}$, als Drg. oder Supp.; Prophylaxe: Betahistin 12 bis $16 \mathrm{mg} 3 \mathrm{x}$ tgl. (Aequamen ${ }^{\circledR}$, Vasomotal ${ }^{\circledR}$ ) alternativ Versuch mit Diuretika Hydrochlorothiazid (Dityde-H ${ }^{\circledR}$; oder Furosemid 20 bis $40 \mathrm{mg} /$ die (Lasix ${ }^{\circledR}$ ), gegebenenfalls intermittierend kombiniert mit Glukokortikoiden (Decortin ${ }^{\circledR}$ $100 \mathrm{mg}$ absteigend); bei therapieresistenten schweren Verläufen: Intratympanale Gentamycin-Instillation; Operation: Saccotomie zur Entlastung des Endolymphsystems; in verzweifelten Fällen: Transtemporale Vestibularisneurektomie, Labyrinthektomie.

\section{Körpergeräusch \\ (Objektiver Tinnitus) \\ und dessen Therapie}

Beim objektiven Tinnitus handelt es sich strenggenommen um keinen Tinnitus: Es finden sich ausschließlich akustische Signale, die vom Organismus des Patienten ausgehen (Körpergeräusch), die vom Untersucher mit dem Stethoskop, z.B. über dem äußeren Gehörgang, gehört werden können. Es lassen sich dabei verschiedene Geräuschformen mit Hinweisen auf die Ätiologie unterscheiden:

- Pulssynchrones Geräusch: Ätiologie: vaskuläre Missbildungen

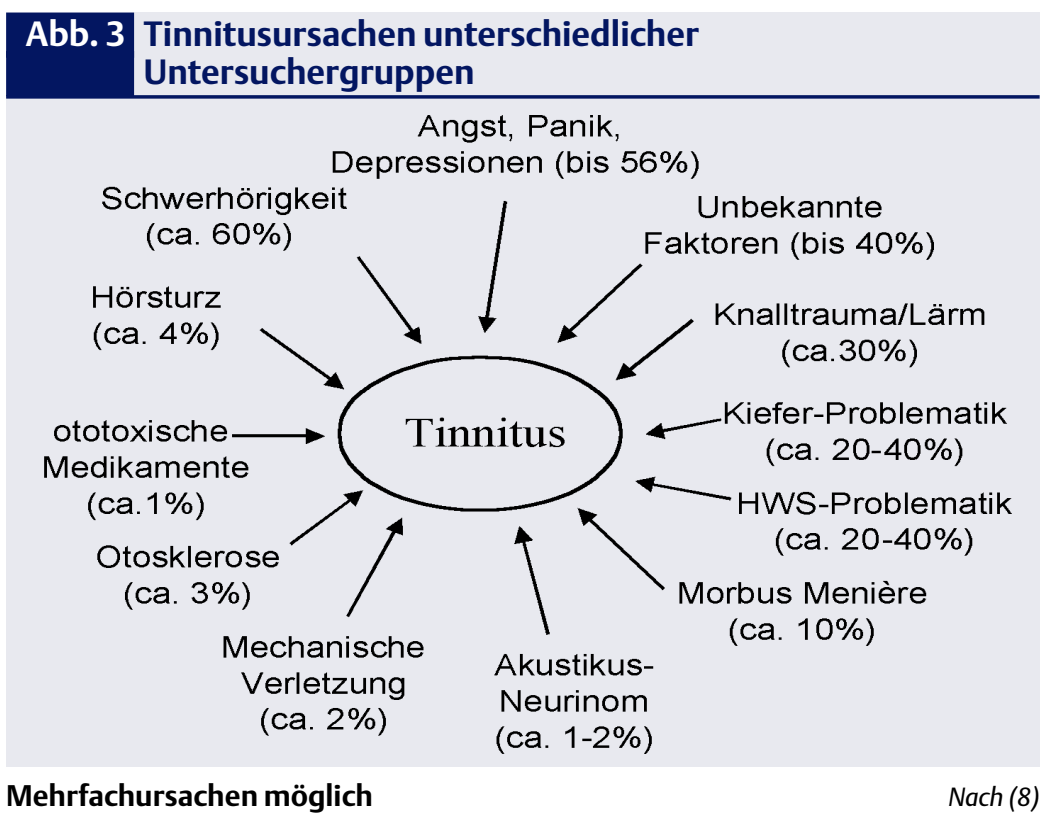

\section{Abb. 4 Endolymphatischer Hydrops bei M. Menière}

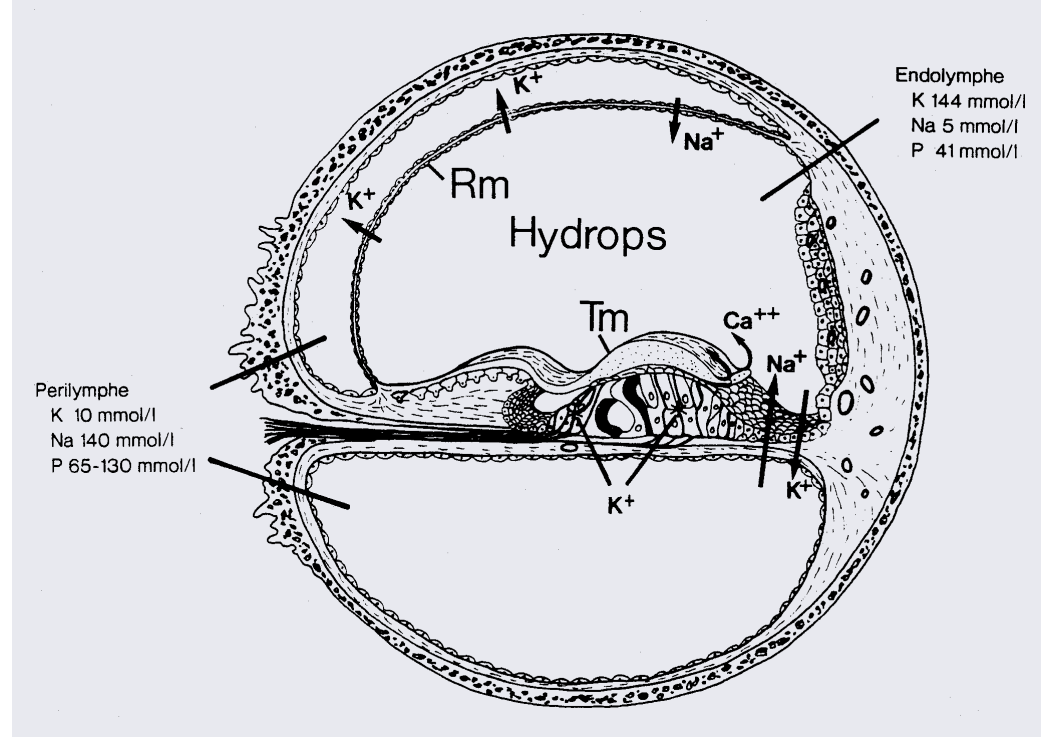

Die Trennmebran zwischen Perilymphe und Endolymphe (ReissnerscheMembran; Rm) wird anfallsweise undicht oder rupturiert, was zu Veränderungen der Kalium-Konzentration der beiden Kompartimente führt

Quelle: Jahnke K. Deutsches Ärzteblatt vom 18.2.94, Heft 7 
- Kontinuierliches Rauschen (verschwindet bei Druck auf die distale Vena jugularis): Ätiologie: venös, häufig einseitig vergrößerter Bulbus jugularis. Kompression durch ein operables Meningeom. Therapie: Ein hochgelegener Bulbus jugularis kann operativ korrigiert werden. Andere venöse Malformationen können endovaskulär revidiert werden. Die Intaktheit der kontralateralen Vena jugularis interna ist vorher zu prüfen. Durch Einlegen eines Ballonkatheters kann der Therapieeffekt zuvor getestet werden

- Serie scharfer Klicks (Dauer mehrere Sekunden bis Minuten): Ätiologie: Tetanische Kontraktionen der Muskeln (Myoklonien) des weichen Gaumens (kann vom Untersucher beobachtet werden) und/oder des Musculus tensor tympani und Musculus stapedius, die auf Läsionen im sogenannten Myoklo- nusdreieck (Nucleus ruber, untere Olive, Nucleus dentatus) zurückgeführt werden. Therapie: Botulinus-Toxin kann in den weichen Gaumen injiziert werden. Auch die operative Durchtrennung des Musculus tensor veli palatini oder des Musculus stapedius ist häufig erfolgreich, sollte aber nur mit zurückhaltender Indikation betrieben werden, da durch Ausfall des Stapediusreflex eine irreversible Geräuschüberempfindlichkeit (Hyperakusis) resultiert. Gelegentlich helfen Carbamazepin (Tegretal ${ }^{\circledR}$, Timonil ${ }^{\circledR}$ )

- Blasendes Geräusch bei der Inund/oder Exspiration: Ätiologie: abnorm weite Tuba auditiva Eustachii. Ursache ist häufig ein erheblicher Gewichtsverlust oder unklare Ätiologie. Therapie: Der Patient ist über die Harmlosigkeit aufzuklären. In Einzelfällen hilft die operative Verengung der Tuba Eustachii. Muskelaufbau-

\section{Tab. 1 Ursachen von Tinnitus}

\section{Mögliche Ursachen Tinnitus}

\section{Mögliche Ursachen Körper-} geräusch (objektiver Tinnitus)

1. Ursachen bei Innenohrerkrankungen

- Lärmschwerhörigkeit

- Knalltrauma

- Morbus Menière

- Hörsturz

- Rundfensterruptur

- Otosklerose

- hereditäre Innenohrerkrankungen

- Ototoxische Innenohrschädigungen

- Durchblutungsstörungen

2. Neurale Ursachen

- Akustikusneurinom

\section{3. zevikogene und} stomatognathogene Ursachen

- Funktionsstörungen der HWS

- Funktionsstörungen des stomatognathen Systems

- Hyperämie bei akuter/ chronischer
1. Extrakranielle Ursachen:

- Fortgeleitete Geräusche einer Halsschlagader

- Aortenklappen-Stenose

- Klicken künstlicher Herzklappen

- Glomus-caroticum-Tumor

- AV-Fistel nach Verletzungen

2. Intrakranielle Ursachen:

- Hämangiom

- Hyperzirkulation bei Anämie Mittelohrentzündung

- Arterio-venöse Fistel nach Schädelverletzungen

- Glomus-tympanicum-Tumor

- Glomus-jugulare-Tumor

- Hochstehender Bulbus venae jugularis

3. Muskuläre Ursachen:

- Spasmus des M. tensor tympani (Mittelohr)

- Spasmus des M. stapedius (Mittelohr)

- Klonus der Schlundmuskulatur

\section{Offene Tuba auditiva}

training mittels gezielter Anpassung einer einseitig erhöhten Aufbissschiene.

In Tabelle 1 sind alle Ursachen von Körpergeräuschen aufgeführt. Es ist nochmals zu betonen: Die Behandlung darf nicht allein durch den Tinnitus begründet werden!

\section{Pseudohalluzination}

Tinnitus wird gänzlich anders generiert, als eine strukturierte akustische Information: Er wird nur in relativ „primitiven“ Formen wahrgenommen (Pfeifen, Rauschen, Brummen etc.). Er ist daher abzugrenzen von akustischen Halluzinationen bei einer Psychose oder von der selten angegebenen organischen „Pseudohalluzination“, bei der Patienten plastische Klanggebilde hören (Kirchenglocken, Melodien, Stimmengewirr etc.), die echoartig nach längerer Einwirkung der jeweiligen Klangbilder noch für Stunden nachhallen oder auch bei starken Emotionen reaktiv auftreten (8). Pseudohalluzinationen sind vermutlich auf Störungen in subkortikalen Bereichen des rechten Temporallappens zurückzuführen (12). Neuroleptika (z.B. Neurocil ${ }^{\circledR} 5$ bis 50 Tropfen) können die Pseudohalluzination innerhalb weniger Minuten beenden (Einschlafhilfe!).

\section{Pragmatische Therapie beim chronischen Tinnitus}

Hier gibt es zwischenzeitlich akzeptierte Therapien, die eine Defokusierung des Tinnitus beabsichtigen und deren Effizienz belegt sind (siehe Beitrag in diesem Heft).

\section{Apparative Therapie}

Viele Tinnituspatienten empfinden eine Erleichterung, wenn äußere Lautquellen den Tinnitus überdecken. Dies kann im einfachen Fall Musik mittels CD oder Tonband abends vor dem Einschlafen sein. In diesem Sinne wird auch Plätschern eines Springbrunnens oder Rauschen eines Ventilators o.ä. eingesetzt. Häufig führen auch Hörgeräte durch verstärkte Wahrnehmung zu einer Tinnitussuppression. Empfohlen werden auch ein- bis zweijährige Behandlungen mit Rauschgenerato- 
ren zusammen mit verhaltenstherapeutischen Ansätzen (7, 8, 9).

\section{Cochleäre Implantate (CI)}

Für Tinnitus-Betroffene mit hochgradiger Innenohrschwerhörigkeit besteht in speziellen Fällen die Möglichkeit, durch Implantation einer elektrischen Sonde in das Innenohr eine Hörfunktion wiederherzustellen, mit der häufig auch eine teilweise Tinnitussupression erreicht werden kann (4). Dieser Eingriff ist nicht geeignet für Betroffene, die noch eine gute Restfunktion des Innenohrs haben, da das Implantat die physiologische Funktion des Innenohrs zerstört.

\section{Gefäßschlingendekompression}

Eine Gefäßschlingendekompression am Eintritt des N. acusticus in den Hirnstamm nach Gardner-Jannetta (analog zur Behandlung der Trigeminusneuralgie) wird durchgeführt (4). Die Indikation ist sehr zurückhaltend zu stellen. Die Operation kann zu einer Hörverschlechterung führen, da der achte Hirnnerv besonders vulnerabel ist. Generell beträgt die Letalität der Operation nach Gardner-Jannetta $1 \%$.

\section{Medikamentöse Therapie}

Hier zeigen sich nur geringe bis fehlende Effektstärken für die meisten der bei Tinnitus propagierten Pharmaka. Infusionen mit Lidocain (Xylocain ${ }^{\circledR}$, Lidocain ${ }^{\circledR}$ ) können zwar zu einer Tinnitusreduktion führen, wegen der erheblichen Nebenwirkungen konnte sich diese Therapie aber nicht durchsetzen. Dies gilt auch für das orale Analogon Tocainide $\left(\right.$ Xylotocan $\left.^{\circledR}\right)(4,7)$.

\section{Unwirksam oder obsolet}

Für die folgenden Medikamente ist eine spezifische Wirkung bei der Tinnitusbehandlung nicht belegt: Carbamazepin, Cinnarizin (Stutgeron $^{\circledR}$ ), Clonazepam (Rivotril ${ }^{\circledR}$ ), Flunarizin (Sibelium ${ }^{\circledR}$ ), Nikotinsäure und -derivate (Niconacid ${ }^{\circledR}$, Roni$\left.\operatorname{col}^{\circledR}\right)$, Meclofenoxat (Helfergin ${ }^{\circledR}$ ), Oxazepam (Adumbran ${ }^{\circledR}$ ), Sulpirid (Dogmatil ${ }^{\circledR}$ ), Pentoxiphyllin (Trental ${ }^{\circledR}$ ), Vitamin A, Ginkgo (3); LaserGinkgo (13).

Auch die Neurektomie wird zur Tinnitusbehandlung allein nicht durchgeführt. Ist aus anderen Gründen (z.B. Tumor) eine Nervendurchtrennung erforderlich, führt dies nur bei $45 \%$ der Patienten zu einem Nachlassen des Tinnitus, bei 55\% bleibt er gleich oder verstärkt sich sogar (4).

Tinnitus is defined as the erroneous perception of sounds and noises in the absence of a relevant external stimulus. It is a common phenomenon that almost everyone has experienced at some time or other. Some $4 \%$ of the German population suffers from chronic noises in the ear/head (tinnitus persisting for more than 1 year), with approximately $15 \%$ of them claiming serious interference with their daily activities (decompensated tinnitus). Clinically, a differentiation is made between subjective and objective tinnitus, the latter being very rare and the sounds are „real“" (due to vascular disorders, palatal myoclonus or an open Eustachian tube, etc.), and may be heard with a stethoscope applied to the head or auditory canal. It is therefore not "true" tinnitus, and is better termed „body noise“ Subjective tinnitus is usually associated with a hearing disorder or is associated with vertigo (Meniere's disease). Only $10-20 \%$ of the patients have no concomitant hearing loss. Options for acute treatment are few. In the chronic stage, treatment measures aimed at coping with the problem must be favoured (e.g. Tinnitus Retraining Therapy, and the use of noise, including the use of hearing aids to achieve a partial masking effect; in cases causing intolerable suffering, hospitalisation in special clinics providing psychotherapeutic treatment is indicated.

\section{Key Words \\ tinnitus - hypacusis - vertigo - pathophysiology - therapy}

\section{Literatur}

1. Bleich $T$, Lamprecht $F$, Lamm H, Jäger B. Der Langzeitverlauf des chronischen Tinnitus. Zeitschrift für Medizinische Psychologie 2001; 2: 79-86

2. Deutsche Tinnitus-Liga (DTL). Tinnitus ist eine Volkskrankheit. Tinnitus-Forum 1999; 3: 58-60

3. Drew J, Davies WE. Gingko biloba in the treatment of tinnitus: Preliminary results of a match-paired, double-blinded placebocontrolled trial involving 1115 subjects. In: Hazell J (ed.). Proceedings of the Sixth International Tinnitus Seminar. Cambridge; Tinnitus and Hyperacusis Center, London 1999: 261-265

4. Feldmann H. Tinnitus. Stuttgart, Georg Thieme Verlag, 1998

5. Fichter M, Goebel G. Psychosomatische Aspekte des chronischen komplexen Tinnitus. Deutsches Ärzteblatt 1996; 93, A1771-A1776

6. Goebel G, Hiller W. Tinnitus-Fragebogen (TF); Ein Instrument zur Erfassung von Belastung und Schweregrad bei Tinnitus (Manual). Göttingen: Hogrefe, 1998

7. Goebel G (Hrsg.). Ohrgeräusche. Psychosomatische Aspekte des chronischen Tinnitus. München, Urban \& Vogel, 2001

8. Goebel G. Tinnitus und Hyperakusis. In: Schulte D, Grawe K, Hahlweg K, Vaitl D (Hrsg.). Fortschritte der Psychotherapie. Göttingen, Hogrefe Verlag, 2003

9. Goebel G. Verhaltensmedizinische Aspekte und Therapie des chronischen Tinnitus. Psychoneuro 2004; 30: 330-336 10. Goebel G, Hiller W. Verhaltensmedizinische Tinnitus-Diagnostik - Eine praktische Anleitung zur Erfassung medizinischer und psychologischer Merkmale mittels des Strukturierten Tinnitus-Interview (STI). Göttingen, Hogrefe Verlag, 2001

11. Hamann K-F. Schwindel aus otologischer Sicht. In: Engelbrecht J, Dietz B. Fortschritt und Fortbildung in der Medizin. Köln, Deutscher Ärzte-Verlag, 2001, 24: 95-99

12. Klostermann W, Vieregge $P$, Kümpf D. Musik-Pseudo-Halluzinose bei erworbener Schwerhörigkeit. Fortschr Neurol Psychiatr 1992; 60: 262-273

13. Lamm K. Rationale Grundlagen einer Innenohrtherapie. Otorhinolaryngol Nova 1995; 5: 153-160

14. Plewnia C, Bartels M, Gerloff C. Transient suppression of tinnitus by transcranial magnetic stimulation. Ann Neurol 2003; 53 : 263-266

15. Zenner HP. Eine Systematik für Entstehungsmechanismen von Tinnitus. HNO 1998; 46: 699-703

\section{Anschrift der Verfasser:}

Priv.-Doz. Dr. med. Gerhard Goebel Facharzt für Psychotherapeutische Medizin Facharzt für Innere Medizin Chefarzt der Medizinisch-Psychosomatischen Klinik Roseneck D-83209 Prien am Chiemsee E-Mail: ggoebel@schoen-kliniken.de Tel: ++49 (0) 8051-68-0

Prof. Dr. med. Ulrich Büttner Ltd. Oberarzt der Neurologischen Klinik der LMU München

Marchioninistr. 15

D-81377 München

E-Mail: ubuettner@nefo.med.uni-

muenchen.de

Tel: ++49 (0) 89 7095-2560 